\title{
Adaptive Spectrum Sensing for Throughput Maximization of Cognitive Radio Networks in Fading Channels
}

\author{
Tae Won Ban, Junsu Kim, and Bang Chul Jung, Member, KIMICS
}

\begin{abstract}
In this paper, we investigate an adaptive cognitive radio $(\mathrm{CR})$ scheme where a sensing duration and a detection threshold for spectrum sensing are adaptively determined according to the channel condition in a fading channel. We optimize the sensing duration and detection threshold of a secondary user to maximize the performance of the secondary user guaranteeing a primary user's secure communication. In addition, we analyze the effect of channel fading on the optimization of the sensing duration and detection threshold. Our numerical results show that the performance of the adaptive $C R$ scheme can be drastically improved if a secondary user can take the advantage of channel information between primary and secondary users.
\end{abstract}

Index Terms - Spectrum sensing, cognitive radio, throughput, Rayleigh fading channel

\section{INTRODUCTION}

IN a cognitive radio (CR) system, there exists a tradeoff between sensing reliability and resource utilization [1-3]. Ghasemi and Sousa [1] showed that there exists an optimum sensing duration to maximize the spectral efficiency of a secondary system. Sensing duration to maximize a secondary user's throughput minimizing the interfering duration was investigated in [2]. Liang et al. [3] also studied the optimal sensing duration to maximize the achievable throughput of a secondary network. In spite of many previous studies, the effect of channel fading was not well investigated although the performance of a CR system can be dramatically improved if we exploit the channel state information in fading channels. In addition, the previous studies relied on the Gaussian approximation of a test statistics.

Based on these motivations, we investigate an adaptive $\mathrm{CR}$ scheme which maximizes the throughput in Rayleigh fading channel by using the exact distribution of the test statistics. We determine the optimal spectrum sensing duration and detection threshold values to maximize a

\footnotetext{
Manuscript received March 3, 2011; revised March 23, 2011; accepted April 6, 2011

Tae Won Ban is with the Mobile Laboratory, KT, Seoul, Korea.

Junsu Kim is with the Department of Electrical and Computer Engineering, University of British Columbia, Vancouver, BC, V6T 1Z4, Canada.

Bang Chul Jung (Corresponding Author) is with the Dept. of Information and Communication Engineering, Collage of Marine Science and the Institute of Marine Industry, Gyeongsang National University, Tongyeong, Gyeongnam, 650-160, Korea (Email: bcjung@gnu.ac.kr)
}

secondary user's throughput efficiency and adapt the values to varying channel condition.

The rest of this paper is organized as follows: In Section 2, we describe a slot structure for CR system and energy detector for spectrum sensing. Then, we propose an adaptive CR scheme and analyze the performance considering the Rayleigh fading channel in Section 3. The numerical results are presented in Section 4.

Finally, conclusions are drawn in Section 5.

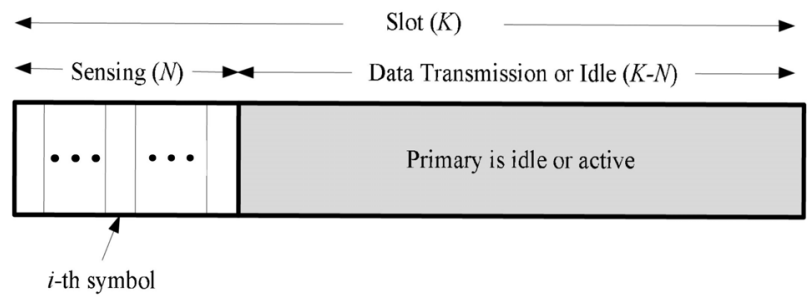

Fig. 1. Slot structure.

\section{SYSTEM MODEL}

\subsection{Cognitive Radio Slot Structure}

Fig. 1 shows a slot structure of the CR system.

A secondary user can opportunistically utilize a primary user's spectrum since it shares a spectrum dedicated to the primary user. Thus, the secondary user can transmit its data only when the primary user does not occupy its spectrum. In order to identify the presence of the primary user, the secondary user attempts to sense the primary user's spectrum. In this paper, we assume that a primary system is time-slotted, one slot is composed of $\mathrm{K}$ symbols, and a secondary user consumes $\mathrm{N}$ symbols to sense a primary user's spectrum. Accordingly, the secondary user can use the remaining $K-N$ symbols to transmit its data if the primary user is idle.

\subsection{Energy Detector}

To identify the presence of a primary user's transmission in a given spectrum band, a non-coherent energy detector is employed because a secondary user is assumed to have no prior knowledge of primary signals which a primary user transmits to its receiver [4]. We assume that a secondary user's sampling rate is the same as the symbol rate of a primary user so that one sample can be obtained for one primary symbol. Then, a spectrum 
sensing can be modeled as a binary hypothesis as follows:

$y(i)=\left\{\begin{array}{cl}n(i) & \mathscr{H}_{0} \text { Primary user is idle } \\ h_{p} x(i)+n(i) & \mathscr{H}_{1} \text { Primary user is active }\end{array}\right.$

where $\mathrm{y}$ is a received signal by a secondary user, $\mathrm{x}$ is a transmit signal of the primary user and satisfies $E_{p}=\mathbb{E}\left[|x|^{2}\right], h_{p}$ is a channel gain from the primary user to the secondary user, $\mathrm{n}$ is an additive white Gaussian noise (AWGN), and $i$ denotes a sampling index. In addition, $n$ is a complex Gaussian random variable with zero mean and variance $N_{0}$, which is denoted by $C \mathcal{N}\left(0, N_{0}\right)$, and $h_{p}$ also follows a complex Gaussian random variable $\sim \mathcal{N}\left(0, \sigma \frac{2}{p}\right)$ since we consider a Rayleigh fading channel. In a slow fading channel, $h_{p}$ remains constant over one slot period but is independent and identically distributed (i.i.d.) across slot periods [5]. Then, we define a test statistic $\mathrm{T}$ to differentiate $\mathcal{H}_{0}$ and $\mathcal{H}_{1}$ as $\mathcal{T} \triangleq \frac{1}{N} \sum \frac{N}{i=1}|\mathcal{Y}(i)|^{2}$.

If $\mathcal{T}$ is smaller than a pre-determined detection threshold $\zeta$, the primary user is declared to be idle. Otherwise, the primary user is declared to be active. The probabilities of detection, and false alarm, which are denoted by $P_{d}$, and $P_{f}$, respectively, are defined as $P_{d} \triangleq \operatorname{Pr}\left[\mathrm{T} \geq \zeta\left|\mathscr{H}_{0}\right|\right]$ and $P_{f} \triangleq \operatorname{Pr}\left[\mathrm{T} \geq \zeta\left|\mathscr{H}_{0}\right|\right]$

\section{ADAPTIVE COGNITIVE RADIO SCHEME}

\subsection{Problem Formulation}

In a CR system, a secondary user's detection probability should be strictly regulated to be greater than a given target value $\hat{P}_{d}$ in order to protect the primary user's communications since misseddetections of a secondary user may cause harmful interference to a primary user. Moreover, there exists a fundamental tradeoff between reliability of spectrum sensing and resource utilization at the secondary user [3]. Thus, we need to optimize both the sensing duration $N$ and the threshold for the hypothesis test $\zeta$ to maximize the secondary user's performance while maintaining the performance requirement of the primary users by satisfying the requirement $\hat{P}_{d}$. In order to measure the secondary user's performance, we define the secondary user's throughput efficiency as the ratio of the secondary user's throughput during data transmission period to the secondary user's average throughput [1].
For given sensing duration and threshold values, the throughput efficiency of a secondary user can be described as

$$
\rho(N, \zeta)=1-P_{f}(N, \zeta)\left(1-\frac{N}{K}\right)
$$

Therefore, the adaptive cognitive radio scheme can be formulated as

$$
\begin{aligned}
& { }^{\max } N \in[1, K] \quad \rho(N, \zeta) \\
& \text { s.t. } P_{d}(N, \zeta) \geq \widehat{P}_{d} .
\end{aligned}
$$

The optimum value of $N$, which is denoted as $N^{*}$, is a solution of the following equation as

$$
N^{*}=\arg \max _{N \in[1, K]} \rho(N, \zeta(N)),
$$

Where $\zeta(N)$ is a detection threshold satisfying the requirement in (2) for a given value of $N$. Therefore, once $N^{*}$ is obtained, the throughput efficiency of the secondary user becomes

$$
\rho^{*}=\rho\left(N^{*}, \zeta^{*}\right)
$$

Where $\zeta^{*}$ denotes the detection threshold value for $N^{*}$. To maximize the throughput and find a corresponding optimal sensing duration $N^{*}$ and threshold $\zeta^{*}$ adaptively, we need to know the false alarm and detection probabilities.

The definition of false alarm probability can be expressed as

$$
P_{f}(N, \zeta)=\operatorname{Pr}\left[\frac{2}{N_{0}} \sum_{i=1}^{N}|n(i)|^{2}>\frac{2 N \zeta}{N_{0}}\right],
$$

Where $\frac{2}{N 0} \sum \frac{N}{i=1}|n(i)|^{2}$ is a chi-square distributed random variable with a degree of freedom $2 N$ denoted by $\mathcal{X} \frac{2}{2 N}$ of which cumulative distribution function (CDF) is given by $F_{\mathcal{X} \frac{2}{2 N}}(x)=\frac{\gamma\left(N, \frac{x}{2}\right)}{\Gamma(N)}[6]$, where $\Gamma(\cdot)$ and $\gamma(\cdot, \cdot)$ denote gamma and lower incomplete gamma functions, respectively. Then, (5) can be rewritten as

$$
P_{f}(N, \zeta)=\frac{\Gamma\left(N, \frac{N \zeta}{N_{0}}\right)}{\Gamma(N)},
$$


Where $\Gamma(\cdot, \cdot)$ denotes an upper incomplete gamma function. In the following subsections, we formulate the detection probability in a slow fading environment to design the adaptive CR schemes in both scenarios which are the case when the channel information is available and vice versa.

\subsection{Proposed Adaptive CR scheme With Channel Information}

The detection probability of the secondary user conditioned on a given $\left|h_{p}\right|^{2}$ can be obtained as

$$
P_{d}\left(N,\left.\zeta|| h_{p}\right|^{2}\right)=\operatorname{Pr}\left[\frac{2}{N_{0}} \sum_{i=1}^{N}\left|h_{p} x(i)+n(i)\right|^{2}>\frac{2 N \zeta}{N_{0}}\right]
$$

Where $\frac{2}{N_{0}} \sum \frac{N}{i=1}\left|h_{p} x(i)+n(i)\right|^{2}$ is a non-central chisquare distributed random variable with a degree of freedom $2 N$ and a non-centrality parameter $\frac{2 N E_{p}\left|h_{p}\right|^{2}}{N_{0}}$, which is denoted by $X_{2 N}^{2}\left(\frac{2 N E_{p}\left|h_{p}\right|^{2}}{N_{0}}\right)$ [7]. The closed-form cumulative distribution function (CDF) of non-central chi-square distributed random variables can be obtained only when the degree of freedom is an even integer [6]. Since $2 \mathrm{~N}$ is an even integer, (6) can be rewritten as

$$
P_{d}\left(N,\left.\zeta|| h_{p}\right|^{2}\right)=Q_{N}\left(\sqrt{\frac{2 N E_{P}\left|h_{P}\right|^{2}}{N_{0}}}, \sqrt{2 N \zeta}\right),
$$

Where $Q_{N}(\cdot, \cdot)$ denotes a generalized Marcum's $Q$ function.

The detection threshold value $\zeta$ can be obtained from (7) for a given value of $N$ and the detection probability requirement $\hat{P}_{d}$. Then, the optimal value of $N$ can be found from (3) and its corresponding throughput efficiency $\rho_{\left|h_{p}\right|^{2}}^{*}$ is also obtained from (4). Finally, the unconditioned optimal throughput efficiency is derived by averaging over $\left|h_{p}\right|^{2}$ as $\rho^{*}=\mathbb{E}_{\left|h_{p}\right|^{2}}\left[\rho_{\left|h_{p}\right|^{2}}^{*}\right]$.

The secondary user can estimate the channel information by exploiting the pilot channel of the primary system [8]. For this operation, the CR user requires prior knowledge about the primary system's pilot channel. For example, if the primary system is a digital TV system, e.g. according to the digital video broadcasting-terrestrial (DVB-T) or the advanced television systems committee (ATSC) standards, the secondary user can be configured to use the corresponding pilot patterns of the primary system to estimate the channel information. This may increase operational complexity and reduce spectral efficiency. However, the complexity can be minimized in slow fading environments taking into account in this paper. Moreover, as will be seen in numerical results, it is possible to obtain a significant performance gain compared to the conventional scheme which does not utilize the channel information.

\subsection{Conventional CR Scheme Without Channel Information}

Since the conventional CR scheme does not exploit the channel information contrary to the proposed scheme, the detection probability requirement $\hat{P}_{d}$ should be satisfied in average sense.

Therefore, the average detection probability can obtained by averaging (7) over $\left|h_{p}\right|^{2}$ as

$$
\begin{aligned}
P_{d}(N, \zeta)= & \mathbb{E}_{\left|h_{p}\right|^{2}}\left[P_{d}\left(N,\left.\zeta N_{0} \zeta h_{p}\right|^{2}\right]\right. \\
= & e^{-\frac{N N_{P}}{N \sigma_{P}^{2} E_{P}+N_{0}}}+\frac{N_{0} e^{-N \zeta}}{N \sigma_{P}^{2} E_{P}+N_{0}} \sum_{n=1}^{N-1} \frac{(2 N \zeta)^{n}}{2^{n} n !} \\
& \Phi\left(1 ; n+1 ; \frac{N \sigma_{P}^{2} E_{P} \zeta}{N \sigma_{P}^{2} E_{P}+N_{0}}\right),
\end{aligned}
$$

Where $\Phi(\because \because \because \cdot)$ denotes the confluent hypergeometric function [9]. The detection threshold for given values of $N$ and $\hat{P}_{d}$ is calculated using (8). Then, the optimal value of $N$ and its throughput also can be obtained as the same procedure to the case with the channel information.

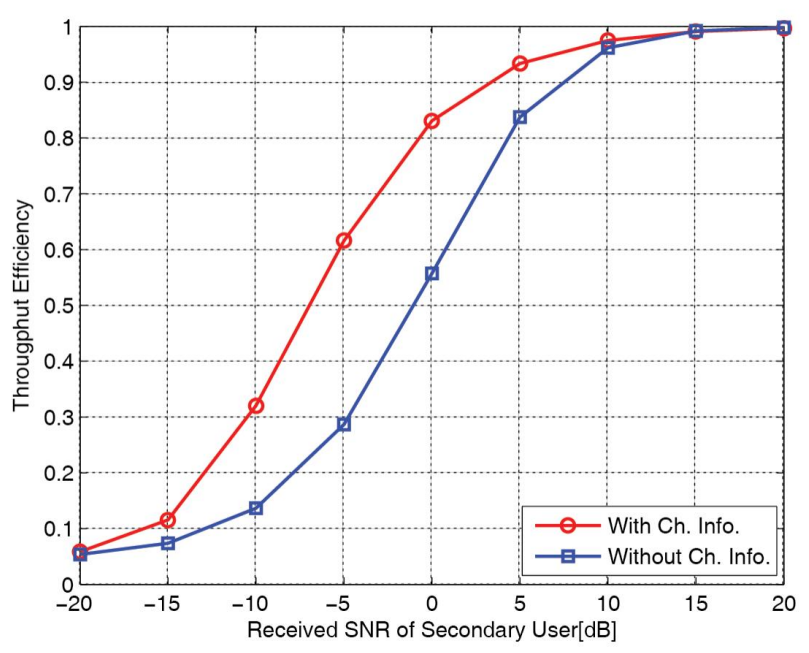

Fig. 2. Throughput efficiency of a secondary user when $\hat{P}_{d}=0.95$. 


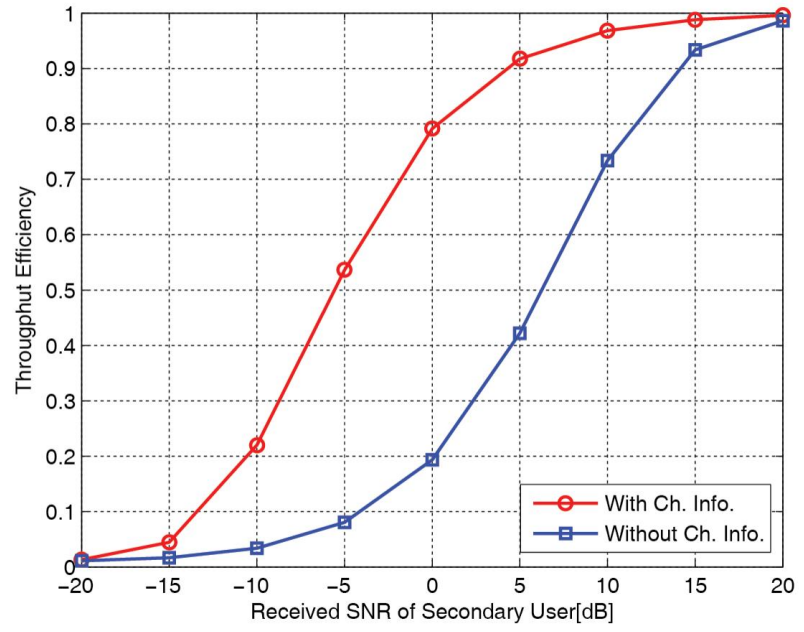

Fig. 3. Throughput efficiency of a secondary user when $\hat{P}_{d}=0.99$.

\section{NUMERICAL RESULTS}

In this section, we evaluate the performance of the proposed adaptive $\mathrm{CR}$ scheme over the conventional CR scheme in terms of throughput efficiency defined in (1).

In the following results, the noise variance $N_{0}$ is assumed to be 1 and then a transmit power value of a primary user $E_{p}$ can be directly translated into a transmit power-to-noise ratio of a primary user without loss of generality. In addition, the average channel gain value $\sigma_{\frac{2}{p}}^{2}$ which can capture the effect of path loss is also assumed to be 1 so that an average transmit power-tonoise ratio of a primary user can be translated into an average received power-to-noise ratio of a secondary user.

Fig. 2 shows the optimal throughput efficiency of a secondary user when the target detection probability of a secondary user $\hat{P}_{d}$ is 0.95 . Solid line with circle markers represents the throughput efficiency for the proposed adaptive CR scheme with channel information and solid line with rectangular markers denotes that for the conventional CR scheme. It is shown that the throughput efficiency of a secondary user increases as the strength of signal to detect increases and the throughput efficiency of the adaptive CR scheme can be greatly improved if the secondary user can exploit the channel information from a primary user. For obtaining the same level of the throughput efficiency, the conventional scheme requires maximally $5 \mathrm{~dB}$. In other words, the proposed adaptive $\mathrm{CR}$ scheme can significantly reduce the transmission power while achieving the same throughput efficiency.

Fig. 3 also shows the optimal throughput efficiency of a secondary user when $\hat{P}_{d}$ is set to 0.99 and all other parameters are the same as in Fig. 2. It is shown that the throughput efficiency of both schemes decreases as $\hat{P}_{d}$ increases. In order to satisfy more strict $\hat{P}_{d}$ value, a secondary user should decrease its detection threshold value and then its throughput decreases because of its false alarm probability increases as the detection threshold value decreases. It is also shown that the adaptive $C R$ scheme which exploits the channel information from a primary user outperforms the adaptive CR scheme which does not exploit the channel information in terms of the throughput efficiency of a secondary user.

\section{CONCLUSIONS}

In this paper, we investigated adaptive CR systems where the sensing duration and the detection threshold are adaptively determined to maximize a secondary user's throughput efficiency in a slow fading channel. We optimized the sensing duration and the hypothesis test threshold values and adapted the values according to varying channel states. Our numerical results show that the benefits of an adaptive $\mathrm{CR}$ scheme can be significantly improved if a secondary user can exploit a channel information from the primary user to the secondary user. In addition, our proposed scheme can strictly protect he primary user from the secondary user compared the conventional scheme because the proposed scheme can always satisfy a given $\hat{P}_{d}$ constraint regardless of channel conditions while the conventional scheme can satisfy the $\hat{P}_{d}$ constraint in an average sense. It should be noted that although the primary user suffers from an extra burden to transmit a pilot for the secondary user, the burden is not significant in a slow fading channel.

\section{ACKNOWLEDGEMENT}

This research was supported by Basic Science Research Program through the National Research Foundation of Korea (NRF) funded by Ministry of Education, Science and Technology (2010-0011140).

\section{REFERENCES}

[1] A. Ghasemi and E. Sousa, "Optimization of spectrum sensing for opportunistic spectrum access in cognitive radio networks," Proc. of IEEE CCNC, pp. 1022-1026, January 2007.

[2] J. Lee, T. Kim, S. Han, S. Kim, and Y. Han, "An analysis of sensing scheme using energy detector for cognitive radio networks," Proc. of IEEE PIMRC, September 2008.

[3] Y. Liang, Y. Zeng, E. Peh, and A. Hoang, "Sensing throughput tradeoff for cognitive radio networks," IEEE Transactions on Wireless Communications, vol. 7, no. 4, pp. 1326-1337, April 2008.

[4] M. Schwartz, W. R. Bennett, and S. Stein, Communication Systems and Techniques. Mcgraw-Hill, 1966.

[5] D. Tse and P. Viswanath, Fundamentals of Wireless Communication. Cambridge University Press, 2005. 
[6] J. G. Proakis, Digital Communications, 4th ed. McGraw Hill, 2001.

[7] H. Urkowitz, "Energy detection of unknown deterministic signals," Proceedings of the IEEE, vol. 55, no. 4, pp. 523-531, 1967.

[8] K. Hamdi,W. Zhang, and K. B. Letaief, "Opportunistic spectrum sharing in cognitive MIMO wireless networks," IEEE Transactions on Wireless Communications, vol. 8, no. 8, pp. 40984109, August 2009.

[9] I. S. Gradshteyn and I. M. Ryzhik, Table of Integrals, Series, and Products, 6th ed. San Diego, CA: Academic, 2000.

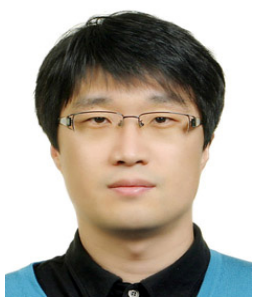

Tae Won Ban received the B.S. and M.S. degrees in electronics engineering from Kyungpook National University, Korea, in 1998 and 2000, respectively, and the Ph.D. degree in electrical engineering from the Korea Advanced Institute of Science and Technology (KAIST), Korea, in 2010.

Since January 2000, he has been working for Korea Telecom, Korea. His research interests include OFDM, MIMO, radio resource management for mobile communication systems, cognitive radio, and relay systems.

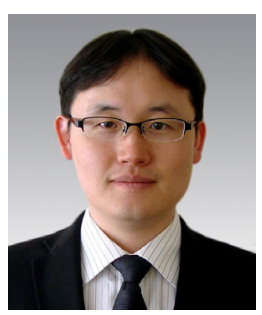

Junsu Kim received the B.S., the M.S., and the $\mathrm{Ph} . \mathrm{D}$. degrees in Electric Engineering and Computer Science from the Korea Advanced Institute of Science and Technology (KAIST), Daejeon, Korea, in 2001, 2003, and 2009, respectively. From March 2009 to August 2009, he was a postdoctoral research fellow at KAIST sponsored by BK21 (Brain Korea 21).

Since October 2009, he has been with the University of British Columbia (UBC), Vancouver, Canada, where he is now a postdoctoral fellow. His research interests include radio resource management, wireless scheduling algorithms, cognitive radio systems, cooperative diversity techniques and physical layer security.

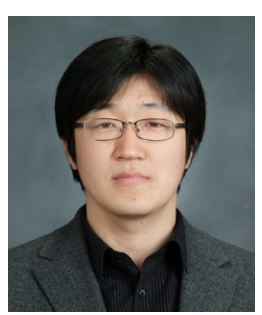

Bang Chul Jung received the B.S. degree in Electronics Engineering from Ajou University, Suwon, Korea, in 2002 and the M.S. and Ph.D degrees in Electrical \& Computer Engineering from Korea Advanced Institute of Science and Technology (KAIST), Daejeon, Korea, in 2004 and 2008, respectively. He was a research professor with KAIST Institute for Information Technology Convergence, Daejeon, Korea, until Feb. 2010. He is now an assistant professor of department of Information and Communication Engineering, Gyeonsang National University, Korea.

Dr. Jung is a member of IEEE, IEICE, Marquis Who's Who in the World (2011 Edition), IBC Top 100 Engineers (2011 Edition). $\mathrm{He}$ was the recipient of the Bronze Prize in Intel Student Paper Contest in 2005, the First Prize in Research Performance Evaluation Sytem (RPES) for Doctorial Student (School of EECS, KAIST) in 2008, the First Prize in KAIST's Invention Idea Contest in 2008, the Bronze Prize in Samsung Humantech Paper Contest in 2009, the IEEE Communication Society Asia-Pacific Outstanding Young Researcher Award in 2011.

His research interests include cognitive radio, cooperative relaying techniques, compressed sensing, physical-layer security, interference alignment, OFDM systems, radio resource management, wireless scheduling algorithm, information theory, link \& system-level simulations for $4 \mathrm{G}$ wireless communication systems, adaptive modulation \& coding, and MIMO systems. 